\title{
PFPRINTE

\section{6) Técnicas projetivas na avaliação psicológica com idosos: Revisão de estudos brasileiros (2000-2018)}

\author{
Eduardo dos Santos de Lima'; Silvana Alba Scortegagna ${ }^{2}$
}

\section{Resumo}

O avanço da expectativa de vida da população incide em diversas áreas do conhecimento, inclusive a de avaliação psicológica com métodos que auxiliam a compreensão psicodinâmica da personalidade. A fim de melhor conhecer esta realidade, este estudo buscou verificar as publicações com o uso de técnicas projetivas na população idosa brasileira. A revisão sistemática baseada na Preferred Reporting Items for Systematic Reviews and Meta-Analysis PRISMA, foi usada para analisar artigos publicados entre 2000 e 2018 no Brasil. As bases de dados foram SciELO, PePSIC, Lilacs, BVS e CAPES. Dos 8 estudos incluídos, os instrumentos mais empregados foram técnicas verbais como, método de Zulliger ( $n=3 ; 37,5 \%$ ), e TAT ( $n=2 ; 25 \%)$. Os estudos com o uso de técnicas projetivas com idosos no Brasil ainda são incipientes, o que deve ser incentivado já que estes instrumentos se mostram importantes ferramentas na avaliação e intervenção, revelando aspectos da estrutura e funcionamento mental.

Palavras-chave: Psicodiagnóstico de idosos; medidas projetivas da personalidade; teste de personalidade; psicologia do idoso; revisão de literatura.

\section{Introdução}

Pela Organização Mundial da Saúde (OMS, 2018) consideram-se idosos os indivíduos com idades a partir dos 60 anos. Entretanto, a passagem para a velhice não é apenas cronológica, mas constitui-se por um conjunto de atributos despertados com o processo de envelhecimento (NERI, 2005). Nesta fase da vida, ocorrem diversas alterações físicas, emocionais, funcionais, socioeconômicas, que podem incidir em condições de maior vulnerabilidade. Os idosos são mais propensos, por exemplo, a desenvolver demência, incluindo a doença de Alzheimer e apresentarem depressão (BYERS et al, 2010), o que requer procedimentos de avaliação psicológica recorrentes.

1 Mestrando do Programa de Pós-Graduação Stricto Sensu em Envelhecimento Humano da Universidade de Passo Fundo. Endereço para correspondência: Universidade de Passo Fundo-UPF, BR 285, São José I Passo Fundo/RS I CEP: 99052-900. E-mail: eduardo_sevn@hotmail.com

2 Professora do Programa de Pós-Graduação Stricto Sensu em Envelhecimento Humano da Universidade de Passo Fundo. E-mail: silvanalba@upf.br 
Os instrumentos de avaliação psicológica são amplamente aplicados em diversos contextos, com amostras predominantemente de crianças, jovens e adultos. Porém, com os idosos ainda não se tem muita clareza do que está sendo realizado com o uso das técnicas projetivas.

Tais técnicas desempenham função essencial na compreensão da estrutura e do funcionamento psicodinâmico da personalidade. Podem revelar dimensões do funcionamento psicológico e conseguir perpassar defesas psíquicas que surgem em situações de autorrelato (FAZENDEIRO; NOVO, 2012). Além disso, técnicas projetivas não dependem de inferências pessoais nem da memória declarativa, pois apresentam estímulos ambíguos ao examinando, como no caso das manchas de tinta, o que facilita a expressão do mundo interior (PINTO, 2014). Isso pode ser especialmente útil com idosos vulneráveis, fragilizados, doentes e sobrecarregados pelas vivências de luto e abandono. $\mathrm{O}$ fato de o idoso ter que contar uma história permite a ele revelar importantes necessidades, preocupações, ansiedades e estilos de enfrentamento, sem ter de abordar o assunto de modo direto. Em muitos casos, seria necessário muito tempo para que, por meio de entrevistas, se construísse uma relação de confiança que mitigasse o uso de suas defesas. Diante do exposto, é necessário verificar a produção científica brasileira sobre a avaliação psicológica de idosos a partir das técnicas projetivas.

\section{Metodologia}

Foi efetuada uma pesquisa bibliométrica, com base no guia de Preferred Reporting Items for Systematic Reviews and Meta-Analyses (PRISMA, 2015), A busca dos estudos foi efetuada nas bases de dados brasileiras SciELO, PePSIC, Lilacs, BVS e CAPES. As palavras-chave utilizadas foram "técnicas projetivas e idosos", "projetivos e idosos", "Rorschach e idosos", "TAT e idosos", "Pfister e idosos", "Zulliger e idosos". Foram incluídos artigos publicados em inglês e português, entre os anos de 2000 a 2018.

A razão da escolha desse período foi para abranger publicações das duas décadas que seguiram-se a partir da resolução no 25/2001 (CFP, 2001) (atualmente revogada) onde foi definido que testes psicológicos são métodos de avaliação privativos do psicólogo Como critérios de elegibilidade foram incluídos apenas artigos publicados no Brasil com a utilização de técnicas projetivas na avaliação de idosos. Excluíram-se estudos que apenas citaram os instrumentos, mas não procederam à avaliação, além de dissertações e teses.

\section{Resultados}

Os resultados das buscas nas bases de dados brasileiras sobre o período de janeiro de 2000 a dezembro de 2018 totalizaram 46 publicações. Aplicando os critérios de exclusão, os estudos selecionados foram encontrados no SciELO $(n=5)$, BVS $(n=2)$, PePSIC $(n=1)$ totalizando 8 estudos, descritos na tabela 1.

Observa-se que nos estudos brasileiros os instrumentos projetivos verbais como o Teste Zulliger $(n=3 ; 37,5 \%)$, e o TAT $(n=2 ; 25 \%)$ foram os mais utilizados, seguidos das técnicas projetivas não verbais como o TPC $(\mathrm{n}=2 ; 25 \%)$ e dos testes gráficos como o HTP $(\mathrm{n}=1$; $12,5 \%)$. As produções se intensificaram entre 2010 e $2017(\mathrm{n}=6 ; 75 \%)$. 
O número amostral variou de 1 a 100 participantes, a média de idade variou de 67,5 a 75 anos. Os estudos tiveram como objetivo avaliar o funcionamento psicológico e da personalidade dos idosos (GIL, 2001; OLIVEIRA et al, 2001; COELHO et al, 2010; DALLAGNOL, 2014); a validade e normatização dos instrumentos (BASTOS-FORMIGHIERI; PASIAN, 2012; GREGOLETI; SCORTEGAGNA, 2017; RIEN et al, 2017) e entendimento do psicodiagnóstico (POZZI; BOFF, 2013).

Tabela 1. Estudos brasileiros com uso de testes projetivos na avaliação de idosos.

\begin{tabular}{|c|c|c|}
\hline Autores (ano) & Objetivo & Técnicas utilizadas / Amostra \\
\hline $\begin{array}{l}\text { Rien.Scortegagna. } \\
\text { et al. ( } 2017)\end{array}$ & $\begin{array}{l}\text { Evidenciar a validade do ZSC } \\
\text { considerando os construtos auto } \\
\text { percepção e relações interpessoais. }\end{array}$ & $\begin{array}{l}\text { ZSC, MEEM / } 30 \mathrm{H} \text { e } 31 \mathrm{M} \text {, idade média de } 71 \text { anos. } \\
\text { Escolaridade média de } 7 \text { anos. }\end{array}$ \\
\hline $\begin{array}{l}\text { Gregoleti. } \\
\text { Scortegagna (2017) }\end{array}$ & $\begin{array}{l}\text { Investigar utilidade do ZSC na avaliação } \\
\text { de idosos com doença renal crônica e } \\
\text { a relação com fatores externos. }\end{array}$ & $\begin{array}{l}\text { ZSC, MEEM e questionário sociodemográfico / } 30 \text { H } \\
\text { e } 30 \text { M, idade média de } 74 \text { anos. Escolaridade média } \\
\text { de } 4 \text { anos. }\end{array}$ \\
\hline $\begin{array}{l}\text { Dallagnol. Schmidt. } \\
\text { Argimon. (2014) }\end{array}$ & $\begin{array}{l}\text { Investigar estados emocionais de ido- } \\
\text { sas participantes de um grupo de con- } \\
\text { vivência. }\end{array}$ & $\begin{array}{l}\text { TAT, MEEM e questionário sociodemográfico / } 10 \mathrm{M} \text {, } \\
\text { idade média de } 75 \text { anos. Ensino fundamental e } \\
\text { médio. }\end{array}$ \\
\hline Pozzi.Boff. (2013) & $\begin{array}{l}\text { Compreender as particularidades e os } \\
\text { desafios de um psicodiagnóstico. }\end{array}$ & $\begin{array}{l}\text { TAT, HTP e WAIS-III / } 1 \mathrm{M}, 75 \text { anos de idade, com } \\
\text { ensino fundamental completo. }\end{array}$ \\
\hline $\begin{array}{l}\text { Bastos-Formigheri. } \\
\text { Pasian. (2012) }\end{array}$ & Elaborar padrões normativos do TPC & $\begin{array}{l}\text { TPC e entrevista semiestruturada / } 38 \mathrm{H} \mathrm{e} 62 \mathrm{M} \text {, idade } \\
\text { média de } 67,5 \text { anos. Escolaridade média } 4 \text { anos. }\end{array}$ \\
\hline Coelho. (2010) & $\begin{array}{l}\text { Identificar se há influência do gênero } \\
\text { e da escolaridade no diagnóstico de } \\
\text { demência. }\end{array}$ & $\begin{array}{l}\text { Zulliger, MEEM, WAIS-III e Lista de REY / } 10 \mathrm{H} \text { e } \\
10 \mathrm{M} \text {. No grupo de H, todos possuíam } 3 \square \text { grau de } \\
\text { escolaridade. No grupo de M a média de escolaridade } \\
\text { era de } 8 \text { anos. }\end{array}$ \\
\hline Gil. (2001) & $\begin{array}{l}\text { Refere-se às relações entre o idoso e o } \\
\text { quadro depressivo. }\end{array}$ & $\begin{array}{l}\text { Questionário Desiderativo, Teste PRIME-MD - } \\
\text { Módulo de Humor e entrevista individual / } 1 \mathrm{He} 5 \mathrm{M} \\
\text { com diagnóstico de depressão. }\end{array}$ \\
\hline $\begin{array}{l}\text { Oliveira, Pasian, } \\
\text { Jacquemin. (2001) }\end{array}$ & $\begin{array}{l}\text { Analisar a vivência afetiva em idosas } \\
\text { em ILPI e não institucionalizadas. }\end{array}$ & $\begin{array}{l}\text { TPC e entrevista semiestruturada / } 50 \text { M, } 25 \text { ILPI e } 25 \\
\text { não institucionalizadas. }\end{array}$ \\
\hline
\end{tabular}

\section{Conclusão}

No Brasil considera-se os estudos sobre as técnicas projetivas em idosos ainda recentes e pouco explorados. Apesar serem encontrados estudos em 2001 (GIL, 2001; OLIVEIRA et al, 2001) ocorre um longo período de tempo onde não são feitas novas pesquisas sobre esse tema. Observa-se um lapso de 9 anos até Coelho et al (2010) retomar o uso de técnicas projetivas como ferramenta de pesquisa. Nesse período houveram mudanças na legislação nacional referente ao uso de testes, as exigências para normatização incluíram largas pesquisas amostrais, porém estas davam preferência a crianças e adultos, deixando o público idoso em espera. Em uma população que está envelhecendo (IBGE, 2018), pesquisas e técnicas que ofereçam possibilidades diagnósticas e sirvam de ferramenta para melhoria da assistência aos idosos devem ser estimuladas, pois segundo Pozzi e Boff (2013), Gregoleti e Scortegagna (2017) instrumentos de avaliação específicos para idosos ainda são escassos. 
Os trabalhos brasileiros mais recentes buscam normatizar os testes e trazer evidências de suas validações dentro do contexto brasileiro para o uso com idosos. Técnicas projetivas requerem estudos com ampla população para serem normatizadas, por isso a importância de diversificar as amostras e incluir a população idosa. No Brasil, segue-se as prerrogativas da resolução n⿳0 09/2018 - CFP (2018), onde, para um teste ser considerado apto para uso profissional por psicólogos, deve apresentar fundamentação e pertinência teórica, definição de objetivos, evidências empíricas, sistema de correção e relatar as características das amostras.

Os estudo que buscaram avaliar os instrumentos enquanto ferramentas de investigação (GIL, 2001; OLIVEIRA et al, 2001, COELHO et al, 2010; DALLAGNOL, 2014) se mostraram eficazes em revelar aspectos significativos das personalidades dos idosos, mostrando a relevância de seus usos enquanto instrumentos de avaliação.

Testes projetivos verbais foram os instrumentos mais utilizados. Sua técnica facilita ao avaliador descobrir aspectos da personalidade dos idosos sem a necessidade de ser invasivo e causar sofrimento por ter de rememorar situações traumáticas.

Cada instrumento oferece informações e possibilidades distintas ao pesquisador. Os estudos concordam que as técnicas projetivas são importantes ferramentas na avaliação e futuras intervenções aos idosos.

\title{
Reconhecimento e agradecimentos
}

O presente trabalho foi realizado com apoio da Coordenação de Aperfeiçoamento de Pessoal de Nível Superior - Brasil (CAPES) - Código de Financiamento 001.

\section{Projective techniques in psychological evaluation with the elderly: Review of Brazilian studies (2000-2018)}

\begin{abstract}
The advancement of the life expectancy of the population focuses on several areas of knowledge, including that of psychological evaluation with methods that help the psychodynamic understanding of the personality. In order to better understand this reality, this study sought to verify the publications with the use of projective techniques in the Brazilian elderly population. The systematic review based on the Preferred Reporting Items for Systematic Reviews and Meta-Analysis PRISMA was used to analyze articles published between 2000 and 2018 in Brazil. The databases were SciELO, PePSIC, Lilacs, VHL and CAPES. Of the 8 studies included, the most used instruments were verbal techniques such as, Zulliger method $(n=3 ; 37.5 \%)$, and TAT $(n=2$; $25 \%$ ). Studies with the use of projective techniques with elderly people in Brazil are still incipient, which should be encouraged since these instruments are important tools in evaluation and intervention, revealing aspects of the structure and mental functioning.
\end{abstract}

Keywords: Psychodiagnosis of the elderly; projective measures of personality; personality test; psychology of the elderly; literature review. 


\section{Referências}

BASTOS-FORMIGHIERI, M.S., PASIAN, S.R. O Teste de Pfister em Idosos, Avaliação Psicológica, Campinas, vol. 11, no. 3, pp. 435-448, 2012.

BYERS, A.L., YAFFE, K., COVINSKY, K.E., FRIEDMAN, M.B., BRUCE, M.L. High occurrence of mood and anxiety disorders among older adults: The National Comorbidity Survey Replication," Arch. Gen. Psychiatry, vol. 67, no. 5, pp. 489-496, May 2010.

COELHO, C.L.M., BASTOS, C.L., CAMARA, F.P., LANDEIRA-FERNANDEZ, J. A influência do gênero e da escolaridade no diagnóstico de demência, Estud. Psicol., Campinas, vol. 27, no. 4, pp. 448-456, 2010.

CONSELHO FEDERAL DE PSICOLOGIA (CFP). Resolução no 25 / 2001, de 30 de novembro de 2001. Brasília, 2001. Disponível em < https://site.cfp.org.br/resolucoes/resolucao-n-25-2001> acesso em 12 de setembro de 2018.

CONSELHO FEDERAL DE PSICOLOGIA (CFP). Resolução no 9 / 2018 de 25 de abril de 2018. Brasília, 2018. Disponível em <http://satepsi.cfp.org.br/docs/Resolu\%C3\%A7\%C3\%A3o-CFP-n\%C2\%BA-09-2018-com-anexo. pdf>, acesso em 12 de setembro de 2018 .

DALLAGNOL, C., BORDIN SCHMIDT, E.I.L.,ARGIMON, I. Estados Emocionais de Idosas a Partir do Teste de Apercepção Temática, Psico, Porto Alegre, vol. 45, no. 1, p. 73, 2014.

FAZENDEIRO, H.M.M., NOVO, R.J.F. Versão Coletiva do Teste de Zulliger Segundo o Sistema Compreensivo de Rorschach, Avaliação Psicológica, Lisboa, vol. 11, no. 3, pp. 407-422, 2012.

GIL, C.A. Contribuições para o diagnóstico compreensivo de pacientes idosos com depressão por meio do Questionário Desiderativo, Psic rev. psicol., São Paulo, pp. 4-25, 2001.

GREGOLETI, V., SCORTEGAGNA, S.A. The Zulliger-CS in elderly on hemodialysis and the relationship between external variables, Paideia, São Paulo, vol. 27, no. 66, pp. 43-50, 2017.

INSTITUTO BRASILEIRO DE GEOGRAFIA E ESTATÍSTICA (IBGE). Projeção da população. Disponível em $<$ https://www.ibge.gov.br/apps/populacao/projecao/> acesso em 12 de setembro de 2018.

NERI, A. As Políticas de Atendimento aos Direitos da Pessoa Idosa expressas no Estatuto do Idoso. A Terceira Idade, São Paulo, vol. 16, no. 34, pp. 7-24, out. 2005.

OLIVEIRA, É.A., PASIAN, S.R., JACQUEMIN, A. A vivência afetiva em idosos, Psicol. Ciência e Profissão, Brasília, vol. 21, no. 1, pp. 68-83, Mar. 2001.

ORGANIZAÇÃO MUNDIAL DA SAÚDE (OMS). Ageing. Disponível em < http://www.who.int/news-room/facts-in-pictures/detail/ageing>, acesso em 12 de setembro de 2018.

PINTO, E.R. Conceitos fundamentais dos métodos projetivos. Ágora Estud. em Teor. Psicanalítica, Rio de Janeiro vol. 17, no. 1, pp. 135-153, Jun. 2014.

POZZI, S.C., BOFF, C. Psicodiagnóstico no idoso e suas particularidades, Contemp. - Psicanálise e Transdiscipl., Porto Alegre, vol. 14, no. jan./dez., pp. 69-86, 2013.

PRISMA. Principais itens para relatar Revisões sistemáticas e Meta-análises: A recomendação PRISMA, Epidemiol. e Serviços Saúde, Brasília, vol. 24, no. 2, pp. 335-342, 2015.

RIEN, M., SCORTEGAGNA, S.A., GRAZZIOTIN, J.B.D.D., BERTOLIN, T.E., RIEN, M., SCORTEGAGNA, S.A., et al. Validity evidence of the Zulliger-CS in older adults with Parkinson's disease, Estud. Psicol., Campinas, vol. 34, no. 4, pp. 560-570, Dec. 2017. 